zum Molekulargewichte dieser Metalle in Natrium. Für das Kalium ist die Löslichkeit in $K O H$ wahrscheinlich gemacht.

Herrn Professor G. Meyer erlaube ich mir an dieser Stelle meinen herzlichsten Dank für die Anregung $\mathrm{zu}$ vorliegender Arbeit auszusprechen, sowie für das rege und fordernde Interesse, welches er stets derselben entgegengebracht hat.

Freiburg i. B., Physikalisch-Chemisches Institut der Universităt, Sommer 1906 bis 1908.

\title{
DIE SPEZIFISCHEN WÄRMEN VON ARGON, WASSERDAMPF, STICKSTOFF, WASSERSTOFF BEI SEHR HOHEN TEMPERATUREN. \\ Von Mathias Pier.
}

(Physikalisch-Chemisches Institut der Universităt Berlin.)

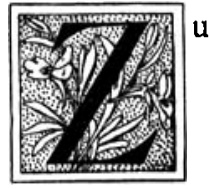
ur Bestimmung der spezifischen Wärmen von Gasen sind drei Methoden im Gebrauch, als erste die Durchströmungsmethode von Regnault zur direkten Messung von $C_{p}$, zweitens die Schallgeschwindigkeit zur Bestimmung von $C_{p}: C_{v}$ und drittens die Explosionsmethode zur Ableitung von $C_{v}$. Letztere wurde von Berthelot und Vieille, Le Chatelier, Langen ${ }^{1}$ ) und dem Verfasser ${ }^{2}$ ) angewendet. Doch ließen die Absolutwerte dieser Methode

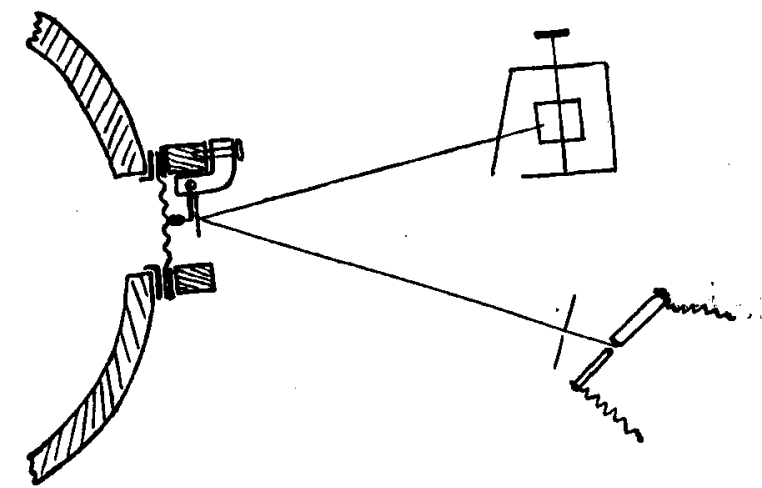

Fig. 280.

noch sehr zu wünschen ubrig, und zwar deshalb, weil die angewendeten Manometer nicht schnell genug den sich mit rasender Geschwindigkeit entwickelten Drucken folgen, dann vermöge ihrer Masse um eine Gleichgewichtslage herumschwingen oder bei Dämpfung das eigentliche Maximum gar nicht mehr anzuzeigen vermögen. Die notwendigen Extrapolationen bedingten große Unsicherheiten der gefundenen Resultate. Aus diesem Grunde wurde in einer früheren Arbeit des Verfassers die spezifische Wärme des Chlors relativ zu derjenigen des als bekannt angenommenen Wasserstoffes bestimmt. Das Streben muBte in erster Linie

I) Mitteilungen aber Forschungsarbeiten, Heft 8, Berlin 1903, Verlag von Springer. - Langen gibt dort genaue Literaturangaben und eine historische $\mathrm{Zu}$ sammenstellung.

a) Zeitschr. f. physik. Chemie 62, 4 (1908), und 66. 6 (1909). darauf gerichtet sein, ein brauchbares MeBinstrument auch für Absolutwerte zu finden. Als solches erwies sich eine gewellte Stahlmembran von etwa $0,1 \mathrm{~mm}$ Dicke und $5 \mathrm{~cm}$ Durchmesser geeignet, deren etwa $\mathrm{I} \mathrm{cm}$ vorstehender Rand zur Befestigung diente. Aehnliche Membranen wurden von Nägel 1) zur Messung der Zündgeschwindigkeiten angewendet. Zur Befestigung wurde sie am Rande zwischen zwei etwa I $\mathrm{cm}$ breite und $3 \mathrm{~mm}$ starke eiserne Ringe gequetscht, damit fest verschraubt und verlötet, so daß ein Nachgeben des Randes unmöglich war. Die Mitte hob sich pro Kilogramm/Quadratzentimeter um etwa o, $\mathrm{r} \mathrm{mm}$ und machte eine Vergrößerung notwendig. Einzig brauchbar erwies sich eine möglichst masselose Spiegelübertragung, die Fig. 280 veranschaulicht.

Auf die Mitte der vertikal mit der Bombe verschraubten Membran ist ein kleiner, oben zugespitzter Messingstift von $3 \mathrm{~mm}$ Hohe und I,2 mm Dicke aufgelötet. Ein $5 \mathrm{~mm}$ breites, I $\mathrm{cm}$ langes und I $\mathrm{mm}$ starkes Messingplättchen, das den Spiegel trăgt und der Membran parallel steht, ist am einen schmalen Ende durch eine mit Spitzen versehene stählerne Achse in schwach konisch ausgehohlten, feststehenden Lagern leicht und sicher beweglich. Als Verbindung mit der Membran und als zweites Gelenk dient ein nur etwa $0,3 \mathrm{~mm}$ langer und o,o I mm starker Neusilberdraht, der einesteils in das andere schmale Ende des beweglichen Hebelarmes, andererseits in den zugespitzten Messingstift eingelötet ist. Als Spiegel diente ein Hohlspiegel aus massivem Silber von $0,6 \mathrm{~cm}$ Durchmesser, der verlötet war, und eine doppelte Brennweite von Ioo $\mathrm{cm}$ hatte. Der maximale Ausschlag betrug allerhöchstens $0,8 \mathrm{~mm}$, und die $z u$ bewegenden Teile wogen insgesamt nicht mehr als I g. Sowohl die Druckkurven, wie die Eichung wurden photographisch aufgenommen auf einen Streifen Bromsilbernegativpapier (Marke N. P. G.), der mit Gummibăndern

I) Näge1, „Versuche uber Zündgeschwindigkeit explosibler Gasgemische "; Mitteilungen tiber Forschungsarbeiten des Ingenieurwesens, Heft 54, Berlin 1908, Verlag von Springer. 
auf einer drehbaren Walze von $64 \mathrm{~cm}$ Umfang festgehalten wurde. Von einer als Lichtquelle dienenden Bogenlampe mit davorgestelltem, $0,2 \mathrm{~mm}$ großem Diaphragma erhielt man einen scharfen Lichtpunkt auf der Walze. Da der Hebelarm mit dem Spiegel I $\mathrm{cm}$ betrug, wurden die Ausschlăge vergrobert im Maßstabe I : roo, also gab I kg/qcm etwa $1 \mathrm{~cm}$ Ausschlag. Die erhaltenen Explosionskurven waren bis zu Temperaturen von $2400^{\circ}$ frei von jeglichen Schwingungen, so daß das Maximum der Explosion wesentlich genauer, als das früher möglich war, ausgemessen werden konnte. Die Kurven waren bei den niedrigsten Temperaturen von $13^{\circ 00}{ }^{0}$ abgeflacht, je höher die Temperatur stieg, um so schneller wurde das Maximum erreicht, um so steiler war der Anstieg, und von etwa $1700^{0}$ erhielt man direkte Spitzen im Diagramm, ein sicherer Beweis, wie schnell das Manometer folgt, während alle früheren, mit anderen Indikatoren erhaltenen Kurven bis zu den höchsten Temperaturen abgerundet aussehen. Nebenstehend sind in Fig. 28I die von Le Chatelier, von Langen und dem Verfasser erhaltenen Kurven zusammengestellt, die mit Knallgas unter Beimengung gleicher Molenzahlen inerter zweiatomiger Gase erhalten wurden. Ersichtlich bekamen jene beiden Autoren zu niedrige Werte des Maximaldruckes. Schwierig gestaltete sich die Eichung, die statisch mit einem Normalinstrument von $\mathrm{Sch}$ ä ffer \& Budenberg vorgenommen wurde.

Wurde nun die Membran auf der Bombe geeicht, indem aus der $\mathrm{CO}_{2}$-Bombe der entsprechende Druck gegeben wurde, was wegen des großen Rauminhaltes von 35 Litern des Explosionsgefäßes nur sehr langsam ging, so zeigte die Membran die Eigenschaften der elastischen Nachwirkung, und zwar um 2 bis $3 \%$ des jeweiligen Ausschlages. Nach einer Explosion und Abkühlung ging das Manometer jedoch genau auf den berechneten Druck zurück, war also innerhalb kurzer Zeiten frei von elastischer Nachwirkung. Um bei der Eichung auch nur kurze Zeit zu belasten, wurde der Membranindikator statt auf die Bombe auf einen kleinen, ausgehohlten Messingblock geschraubt, dessen nur etwa $20 \mathrm{ccm}$ großer Hohlraum mit Paraffinöl gefüllt war und mittels eines Dreiweghahnes entweder mit der Atmosphäre oder dem Vergleichsinstrument und der $\mathrm{CO}_{2}$-Bombe kommunizierte. Durch Drehen des Dreiweghahnes konnte also der Indikator schnell belastet und entlastet werden, während das Vergleichsinstrument dauernd belastet blieb. Auf diese Weise wurde sowohl mit Fernrohr und
Skala die Abhängigkeit der elastischen Nachwirkung von der Zeit bestimmt, indem in kurzen Zeitintervallen der jeweilige Ausschlag abgelesen und graphisch auf die Zeit o extrapoliert wurde, andererseits wurde auch Nullinie, wie Anstieg und Ausschlag photographisch registriert mit der in Fig. 28o skizzierten Einrichtung. Es ergab sich auf beide Weisen übereinstimmend, daß erst nach etwa 5 Sekunden die elastische Nachwirkung eintritt.

Die sonstige Einrichtung war dieselbe, wie in der früheren, oben angegebenen Arbeit beschrieben wurde. Als Explosionsraum diente eine stählerne Hohlkugel von 35 Liter. Inhalt, die jetzt nur zwecks genauer Anfangstemperatur in einem großen, mit Rührer und Thermometer versehenen Wasserbade saB. Gezündet wurde genau in der Mitte durch einen elektrischen

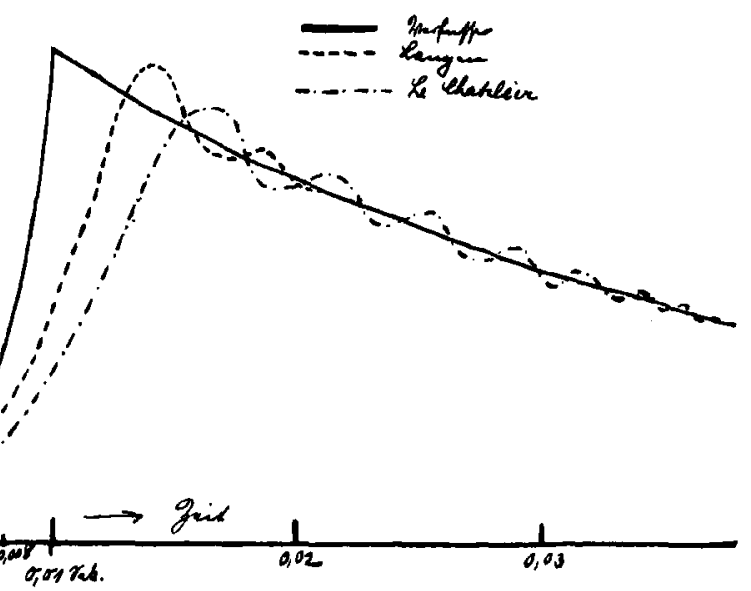

Fig. 28r.

Funken. Die Bombe hatte zwei kreisrunde Oeffnungen von $5 \mathrm{~cm}$ Durchmesser auf beiden Seiten; auf die eine wurde die Indikatorvorrichtung vermittelst Winkeleisen und Schrauben, mit Bleidichtung versehen, befestigt; auf der entgegengesetzten Seite saB eine Verschraubung zur Aufnahme der Zündung und eines Viktoriaventils zum Füllen und Verschließen. Als explosibles Gasgemisch kam reines, elektrolytisch dargestelltes Knallgas zur Anwendung, das in 2o hintereinandergeschalteten eisernen Zellen, deren Wandungen als Kathoden, während isoliert eingeführte Nickelelektroden als Anoden dienten, aus sechsprozentiger Natronlauge mit I Io Volt und I5 Amp. dargestellt wurde. Die außerordentlich starke Knallgasentwicklung gestattete die Füllung innerhalb einiger Minuten. Dazu wurden nun verschiedene Mengen von an der Explosion nicht teilnehmenden Gasen, wie $\mathrm{N}_{2}, \mathrm{H}_{2}, \mathrm{~A}, \mathrm{O}_{2}$, zugesetzt, die die Temperatur variierten, und deren spezifische Wärme bestimmt werden sollte. Die.Mengenverhältnisse der einzelnen, feucht eingefüllten Gase wurden aus ihren Partialdrucken an einem Quecksilber- 
manometer mit Glasskala und Fernrohr abgelesen. Nachdem die Bombe mit der Wasserstrahlluftpumpe bezw. Quecksilberluftpumpe evakuiert war, wurden zuerst die inerten Gase und dann Knallgas eingefüllt, so weit, dab der Anfangsdruck vor der Explosion immer I Atmosphäre betrug. Zwischen Fullung und Explosion wurde mindestens $1 / 2$ Stunde gewartet, um den Gasen Zeit zur Mischung und Annahme der Wasserbadtemperatur zu lassen; ebenso lange wurde nachher gewartet, um den Unterdruck nach Verbrennung und Abkuhlung zu messen, der von demjenigen vor der Explosion um höchstens $0, \mathrm{r} \%$ abwich.

Diagramm $\tau$.

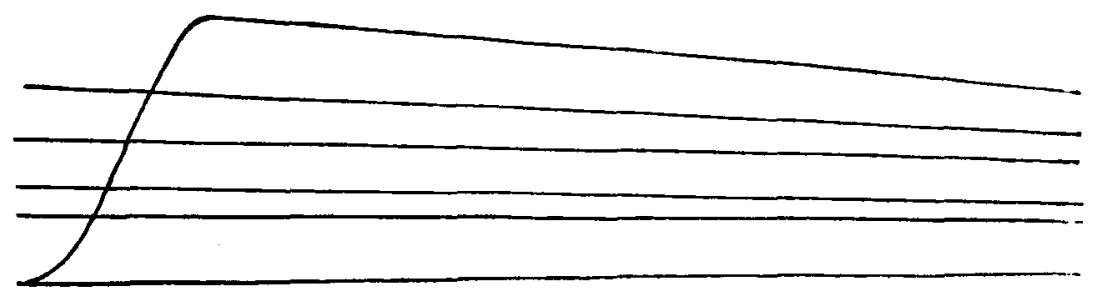

Diagramm 2.

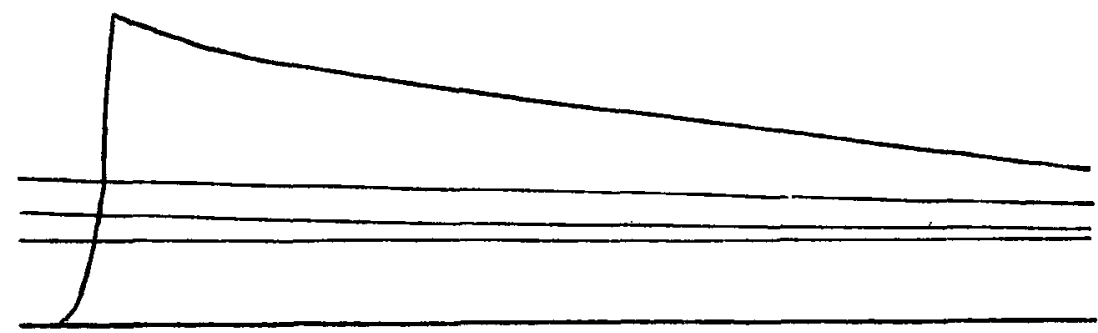

Diagramm 3.

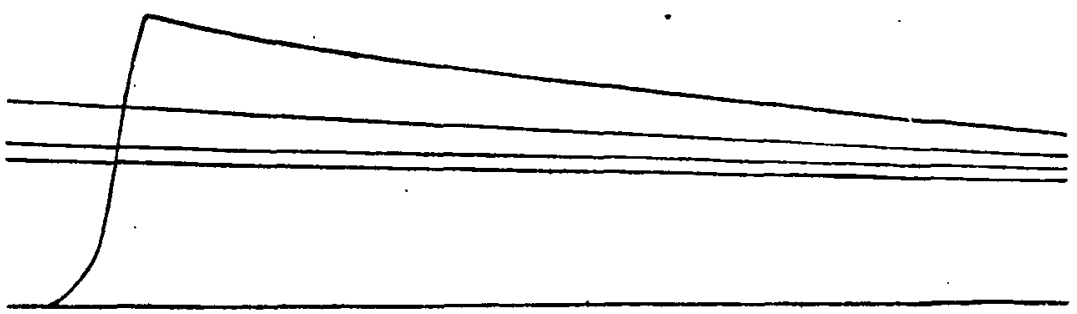

Fig. 282.

Zur Berechnung gilt die Formel:

(I) $Q=t_{2}\left(C_{H_{2} O}^{\prime \prime}+m_{i} C_{i}^{t_{2}}\right)-t_{1}\left(C_{H_{2} O}^{\prime} O+m_{i} C_{i}^{\prime}\right)$, worin bedeuten:

$Q=$ Wärmetornung der Reaktion bei $t_{1}$ und konstantem Volum $(57290 \mathrm{Kal}$. für I Mol $\mathrm{H}_{2} \mathrm{O}$ ), [= Bildungswärme $\mathrm{H}_{2} \mathrm{O}$ flüssig $=682001 \% .-$ Verdampfungswärme $=18$. $590,-290=$ Arbeit derAtmosphäre];

$t_{1}=$ Temperatur vor der Explosion;

$t_{2}=$ Maximaltemperatur der Explosion;

I) Nernst: "Ueber die Berechnung elektro. motorischer Kräfte aus thermischen Größen "; Sitz.- Ber. d. Königl. Preuß. Akad. d. Wiss. 1909, VIII, 254.
$C_{H_{2} O}=$ mittlere Molekularwärme von Wasserdampf zwischen $0^{0}$ und $t_{1}$ resp. $t_{2}$;

$C_{i}=$ mittlere Molekularwärme der zugesetzten Gasmasse zwischen $0^{0}$ und $t_{1}$ resp. $t_{2}$;

$m_{i}=$ Anzahl Mole zugesetzter Gasmasse pro Mol $\mathrm{H}_{2} \mathrm{O}$.

$\mathrm{Da}$ wir bei konstantem Volum explodieren lassen, ist nach den Gasgesetzen die Temperaturerhőhung proportional der Drucksteigerung:

oder

$$
\frac{273+t_{2}}{273+t_{1}}=\frac{P}{p \cdot \varepsilon}=\frac{\pi}{\varepsilon}
$$

2. $\quad t_{2}=\left(\frac{\pi}{\varepsilon}-\mathrm{I}\right)\left(273+t_{1}\right)+t_{1}$,

wenn bedeutet:

$P=$ Maximaldruck der Explosion,

$p=$ Anfangsdruck der Explosion (nahe gleich I Atmosphäre),

$\pi=$ Drucksteigerungsver. hältnis,

$\varepsilon=$ Verhăltnis der Molekulzahl nach der Explosion zu derjenigen vor derselben.

Die Aufnahme der Explosion erfolgte nun in der Art, daß die Walze clurch einen Elektromotor in Umdrehung versetzt wurde, und zwar machte sie in der Sekunde zwei Umdrehungen, so daB $128 \mathrm{~cm}$ der Abszisse des Diagramms I Sekunde entsprechen; dann offnete man den Kasten, ließ sich die Nulllinie markieren und explodierte; nach einigen Sekunden wurde die Kiste wieder geschlossen. Es wurde immer das Maximum des Explosionsdruckes ohne jede Extrapolation zur Berechnung benutzt.

\section{A. Versuche mit Argon.}

(Diagramm I, Fig. 282.)

Auf Vorschlag von Herrn Professor Nerast wurden zur Prüfung der Methode Versuchc mit einem einatomigen Gase ausgeführt, denn bekommt man hierfür den von der kinetischen Gastheorie verlangten Wert ${ }^{1}$ ): $C_{v}=$ konstant = 2,98I, dann gibt die Explosionsmethode $\mathrm{Ab}$ solutwerte. Benutzt wurde reines Argon, das uns in einer Quantität von 30 Litern Herr Pro-

I) Vergl. Nernst, Theoretische Chemie, 6. Aufl., Ig09, S. 206 a. 207 . 
fessor Franz Fischer nach seiner Methode aus $\mathrm{CaC}_{2}$ darstellte. Das Verhaltnis Wasserdampf zu Argon wurde noch variiert durch Erhohung der Anfangstemperatur auf $53^{\circ}$, wie die Versuche I5I bis I 55 zeigen. Bei sämtlichen Explosionen wurde ein kleiner Ueberschuß von $H_{2}$ zugesetzt, um die Dissoziation des Wasserdampfes unmerklich zu machen.

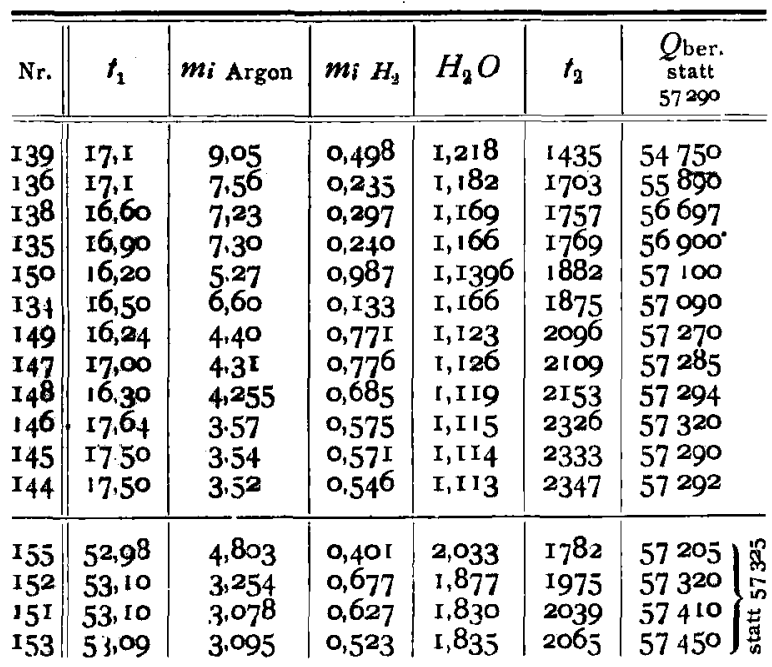

Hieraus berechnet sich mit Gleichung (I) unter ausgezeichneter Uebereinstimmung für Wasserdampf im Mittel, gültig zwischen $0^{0}$ und 23: $0^{0}$ :

$C v_{m H_{2} O}=6,065+0,0005 t+\frac{0,2}{10^{9}} t^{3}$

und für Argon praktisch der theoretische Wert:

$$
C v=2,977 \text {. }
$$

Die aus diesen Formeln nach Gleichung (1) berechneten Wärmetönungen sind in der letzten Spalte der Tabelle verzeichnet. Eine Abweichung ist nur bei niederen Temperaturen vorhanden, wo das Maximum sich langsam einstellt und demgemäB schon Verluste eingetreten sind.

\section{Versuche mit Stickstoff.}

(Diagramm 2, Fig. 282.)

Hierzu wurde Bombenstickstoff ${ }^{1}$ ) verwendet, der nach einer Explosion mit $H_{2}$ im Ueberschuß rein war. $Z u$ bemerken ist, daß hier zwei verschieden starke Membranen von verschiedener Empfindlichkeit angewendet wurden, die genau

1) Bombenstickstoff ist mit einigen Prozenten Sauerstoff verunreinigt. Für vorliegenden Zweck konnte er also einfach gereinigt werden durch eine Explosion von Knallgas mit einem Ueberschuß an Wasserstoff. Letzterer wurde ja ohnehin zugesetzt, um die Dissoziation des Wasserdampfes zurïckzudrängen. Maßgebend für die Berechnung war in diesem Falle natürlich die nach dem Versuche am Quecksilbermanometer abgelesene Kontraktion, die dem gebildeten Wasser entspricht. dieselben Resultate ergaben. Auch hier wurde die Wasserdampfmenge variiert.

\begin{tabular}{l|l|l|ll|l|l|l|l|l|l}
\hline \hline Nr. & $t_{0}$ & mi & $N_{2}$ & $m_{i} H_{2}$ & $t_{\mathrm{a}}$ & $H_{2} O$ & $\begin{array}{c}Q \text { ber. } \\
\text { statt } \\
57290\end{array}$ \\
\hline
\end{tabular}
a) Membran I.

\begin{tabular}{|c|c|c|c|c|c|}
\hline 74 & $2 \mathrm{I}, \mathrm{O}$ & 6,596 & I254 & $\mathrm{I}, 207$ & 55100 \\
\hline $9 \mathrm{I}$ & 18,3 & 5,709 & I394 & $\mathrm{I}, 164$ & 55200 \\
\hline 90 & 20,6 & 5,643 & I. 406 & $\mathrm{r}, 17^{\mathrm{i}}$ & 55240 \\
\hline 92 & I9 I & 5,209 & 1504 & $I, r_{42}$ & $5597^{\circ}$ \\
\hline 93 & 20,0 & $4.5 \mathrm{I} 4$ & 1657 & I, I 39 & 370 \\
\hline 94 & 20,8 & 3.902 & $18 \mathrm{I}_{4}$ & $\mathrm{I}, \mathrm{I} 3^{8}$ & $5^{6800}$ \\
\hline 95 & $2 I, 5$ & 3,235 & 2018 & I, 126 & 57240 \\
\hline 9 & 19.5 & 2,833 & 2155 & $I, 109$ & 57295 \\
\hline 97 & $22, I$ & 2,6 I9 & 2230 & $\mathrm{I}, \mathbf{I} 10$ & $573^{10}$ \\
\hline 15 & $23, I$ & 2,282 & $235^{8}$ & I,097 & 57315 \\
\hline & $2[, 3$ & 2,162 & $243^{6}$ & 1,062 & $5735^{\circ}$ \\
\hline & 17,0 & $1,45^{6}$ & 2735 & $1,05^{8}$ & 57500 \\
\hline & 78 & $I, 164$ & $287 \mathrm{I}$ & I, 054 & $5745^{\circ}$ \\
\hline
\end{tabular}

b) Membran 2.

\begin{tabular}{|c|c|c|c|c|c|}
\hline 16,85 & 5,806 & $0,47^{6}$ & $13^{17}$ & $I, 14 \mathrm{I}$ & 55490 \\
\hline 17,09 & 4,799 & 0,546 & 1492 & 1,130 & $5^{6} 310$ \\
\hline 16,60 & 3.718 & 0,517 & $175^{2}$ & $\mathrm{x}, 1 \mathrm{IO}_{5}$ & 57100 \\
\hline$I 6,8 \mathrm{I}$ & 3,186 & 0,443 & 1917 & 1,096 & 57240 \\
\hline $\mathrm{I} 7,08$ & 2,544 & 0,366 & 2144 & $\mathrm{r}, 086$ & 57297 \\
\hline 17,11 & I, 894 & 0,534 & 2325 & $\mathbf{I}, 077$ & 57300 \\
\hline $\begin{array}{l}52,98 \\
53,06\end{array}$ & $\begin{array}{l}3,966 \\
1,984\end{array}$ & $\begin{array}{l}0,95^{2} \\
0.35^{6}\end{array}$ & $\begin{array}{l}13^{88} \\
2078\end{array}$ & $\begin{array}{l}2,003 \\
1,636\end{array}$ & 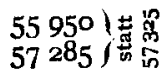 \\
\hline
\end{tabular}

Setzen wir für $\mathrm{H}_{2} \mathrm{O}$ die oben erwähnte Formel, so berechnet sich für Stickstoff im Mittel:

$$
C v_{m N_{3}}=4,900+0,00045 t \quad \text { III. }
$$

In der letzten Spalte sind wieder die berechneten $Q$-Werte verzeichnet.

\section{Versuche mit Wasserstoff. \\ (Diagramm 3, Fig. 282.)}

Zur Verwendung kam Bombenwasserstoff. Die Kurven verliefen wesentlich steiler, als diejenigen von Stickstoff; noch größer jedoch war der Unterschied gegenüber den Argonkurven; diese verschiedene Zünd- wie $\mathrm{Ab}$ kühlungsgeschwindigkeit ist bedingt durch das verschiedene Wärmeleitvermögen der Gase, das

\begin{tabular}{|c|c|c|c|c|c|}
\hline Nr. & $t_{1}$ & $m_{i H_{3}}$ & $\mathrm{H}_{2} \mathrm{O}$ & $t_{2}$ & $\underset{\text { statt }}{Q} \begin{array}{l}Q 7293 \\
\text { ber. }\end{array}$ \\
\hline I6 & 17,73 & 5.849 & $\mathrm{I}, 15^{\mathrm{I}}$ & 1417 & 55600 \\
\hline 165 & 18,00 & 5,044 & I, I34 & I 592 & 56800 \\
\hline 166 & 16,13 & 4,057 & I, rör & I8 31 & 57 I92 \\
\hline 167 & 16,39 & 3.398 & 1,092 & 2017 & 57268 \\
\hline 168 & 16,98 & 2,683 & I,086 & $225^{\circ}$ & 57290 \\
\hline
\end{tabular}
mit dem Molekulargewicht bekanntlich abnimmt.

Für Wasserstoff berechnet sich hieraus im Mittel:

$$
C v_{m H_{2}}=4,700+0,00045 t \quad \text { IV. }
$$

Die mittleren Molekularwärmen zwischen o und $13^{\circ 0}{ }^{\circ}$ resp. o und $25^{\circ 0}$ ") von Argon, Wasserdampf, Stickstoff und Wasserstoff dürften hiermit auf vielleicht kleine Bruchteile von Pro- 
zenten sichergestellt sein. Ausgezeichnet zur Bestimmung der spezifischen Wärme von Gasen eignet sich die Explosionsmethode von 1600 bis $2300{ }^{0}$; bei niedrigeren Temperaturen ist eine Korrektion notwendig, bei hơheren verhindern die heftigen Erschütterungen und die Eigenschwingungen der MeBinstrumente vorläufig noch eine genaue Messung.

Zum Vergleich sind nachstehend die Molekularwärmen bei konstantem Druck angegeben, wie sie nach der gebräuchlichsten Methode, der Durchströmungsmethode von Holborn und Hennig ${ }^{1}$ ), bestimmt wurden.

I. Stickstoff, $C_{p}$-Mittel zwischen $20^{0}$ und $t$.

\begin{tabular}{c|c|c}
\hline$t$ & $\begin{array}{c}C_{p \text { beob. }} \\
\text { Holborn } \\
\text { und Hennig }\end{array}$ & $\begin{array}{c}C_{p} \text { ber. } \\
=6,885+0,00045 t\end{array}$ \\
\hline 200 & 6,720 & 6,984 \\
440 & 6,773 & 7,092 \\
630 & 6,899 & 7,178 \\
800 & 6,992 & 7,254 \\
844 & 7,031 & 7,274 \\
1000 & 7,238 & 7,344 \\
I181 & 7,210 & 7,425 \\
1347 & 7,300 & 7,500
\end{tabular}

Die Abweichung ist durchgängig etwa $4 \%$; doch müssen auch nach den $C_{p} / C_{v}$-Bestimmungen die Holbornschen Zahlen zu klein sein; es ergibt sich ferner für Luft und für Sauerstoff übereinstimmend, also auch für Stickstoff, aus den direkten Messungen Regnaults, wie aus der Schallgeschwindigkeit die Molekularwärme bei Zimmertemperatur zu 6,9 anstatt zu 6,7 nach Holborn.

$\mathrm{Zu}$ den Holbornschen Zahlen ist zu bemerken, daB dieselben, wie es in Spalte 3 geschehen ist, einmal wegen der Abweichung des Wasserdampfes vom idealen Gaszustande ${ }^{2}$ ), andererseits wegen der aber $1200^{\circ}$ beginnenden Dissoziation ein klein wenig verkleinert werden müssen, um sie mit den meinigen vergleichbar zu machen. Beide Korrektionen sind übrigens, wie die Zahlen der dritten Spalte zeigen, nur klein. Die Uebereinstimmung ist praktisch vollkommen.

I) Drudes Ann. 23, 842 (1907).

2) Näheres daraber in einer demnächst erscheinenden Arbeit des Herrn Levy.
2. Wasserdampf, $C_{p}$-Mittel zwischen r ro ${ }^{\circ}$ und $t$.

\begin{tabular}{c|c|c|c}
\hline$t$ & $\begin{array}{c}C_{p} \text { beob. } \\
\text { Holborn } \\
\text { und Hennig }\end{array}$ & $\begin{array}{c}C_{p} \text { korrigiert } \\
\text { Holborn } \\
\text { und Hennig }\end{array}$ & $\begin{array}{c}C_{p} \text { ber. } \\
=8,050+0,0005 t \\
+\frac{0,2}{10^{8}}\end{array}$ \\
\hline \hline 270 & 8,359 & 8,197 & 8,246 \\
440 & 8,493 & 8,363 & 8,348 \\
620 & 8,500 & 8,435 & 8,473 \\
800 & 8,608 & 8,560 & 8,623 \\
820 & 8,796 & 8,749 & 8,642 \\
834 & 8,678 & 8,632 & 8,656 \\
1000 & 8,931 & 8,894 & 8,830 \\
I183 & 9,082 & 9,051 & 9,062 \\
1200 & 9,259 & 9,209 & 9,086 \\
1327 & 9,387 & 9,336 & 9,283 \\
1350 & 9,511 & 9,460 & 9,316
\end{tabular}

Nägel hat in der früher erwähnten Arbeit bei der analytischen Untersuchung der Zündungsfortpflanzung berechnet, daB an der kugelförmigen Bombenwand bei zentraler Zündung die Gase durch adinbatische Kompression auf einige hundert Grad vorgewărmt seien. Dann wären die Druckmessungen für die Berechnung der spezifischen Wärmen gar nicht anwendbar. Demgegenüber ist zu betonen, daß bei dieser Rechnung der Wärmeaustausch im Gase selbst vernachlässigt ist, daß aber vor allem durch die plötzliche Drucksteigerung der ganze Inhalt höchstwahrscheinlich vollkommen durcheinander gewirbelt wird, daß man also den Vorgang wohl nicht in einzelne Phasen zerlegen darf. Dementsprechend ist an den Kurven nichts davon sichtbar; man könnte ferner für Argon unmöglich den von der kinetischen Gastheorie verlangten Wert erhalten, wenn obige Fehlerquelle von Belang wäre. Auch die sonstigen Versuchsresultate lassen nirgends derartige Störungen erkennen.

Herrn Professor Nernst bin ich für das dauernde und lebhafte Interesse an der Arbeit zu großem Dank verpflichtet.

Herrn Professor Franz Fischer danke ich verbindlichst für die Darstellung der großen Mengen Argon 1).

(Eingegangen: 26. Juni.)

I) Eine ausführliche Beschreibung der Versuche erfolgt derinächst an anderer Stelle.

\section{DIE KOLLOIDSTOFFE IN DEN TONEN. Von Paul Rohland.}

Das Problem der Kaolinisierung ist bisher im wesentlichen nur von geologischer Seite in Angriff genommen; und zwar geschah dieser Angriff von zwei Punkten aus.

Die eine Partei, deren Hauptvertreter Weinschenck ist, nimmt den Standpunkt ein, daß ihrer Ansicht nach postvulkanische Prozesse, pneumatolitische und pneumahydatogene, bei denen kaolinisierende Agentien in Form von Dămpfen und heißen Lösungen von Fluor-BorSchwefelverbindungen auftraten, die Kaolinisierung herbeigefuhrt haben. 